\title{
La neología política peruana en el nodo UNIFE del Observatorio de Neología (Universidad Pompeu Fabra) - periodo 2003-2019
}

\section{Peruvian Political Neology in the UNIFE Node of the Observatory of Neology (Pompeu Fabra University) - Period 2003-2019}

\author{
Rosa Luna García \\ UNIFE \\ rosalunag@unife.pe
}

\begin{abstract}
Resumen
El objetivo central de este estudio, de corte empírico, aplicado, documental y terminográfico sistemático fue determinar la productividad de los neologismos políticos peruanos recogidos en el nodo peruano unife del Observatorio de Neología de la Universidad Pompeu Fabra durante el periodo 2003-2019. La investigación arribó a las siguientes conclusiones generales: 1) El escaso número de tokens (repeticiones) del corpus neológico político peruano podría atribuirse a problemas en la detección asistemática, a la movilidad de los neólogos que integran el nodo de Antenas Neológicas Unifé o a la variabilidad del sentimiento neológico. Las autoras Adelstein y Freixa (2013) argumentaron en una investigación realizada a partir de datos proporcionados por el Observatorio de Neología de la Universidad Pompeu Fabra que estas plataformas se caracterizan por la presencia de un elevado número de neologismos con baja estabilización. 2) En lo que respecta a la productividad anual de neologismos políticos se observó una tendencia bastante equilibrada entre los dos octenios de estudio. El primer octenio del corpus de estudio (2004-2011) alcanzó un $42,2 \%$ frente a un 57,6 \% del segundo, con un 15,2 \% de diferencia entre ellos. 3) Se constató que los criterios utilizados para la diccionarización de neologismos en diccionarios de lengua general (exclusión de neologismos locales, efímeros, derivados de nombres propios y de palabras con sufijos productivos transparentes [Aldenstein y Freixa, 2013]), diferían de aquellos relacionados con lo de naturaleza geolectal. Son varios los autores que demuestran que en estos últimos priman factores como el carácter testigo de los nuevos vocablos en un determinado periodo político (Matoré, 1953; Aldenstein y Freixa, 2013) o la necesidad de denominar una palabra nueva (Barnhart, 2007), 4) Los diarios formales El Comercio y La República fueron los más productivos en neología política peruana concentrada básicamente en las secciones política, actualidad, nacional, página editorial y columnas. 5) En la acuñación de neologismos políticos peruanos se observó que la derivación, sufijación y prefijación, conjuntamente con la composición (patrimonial y culta) alcanzaron un elevado 81,7 \%. Este resultado se confirma en diversos trabajos sobre neología política (Díaz, 2001; Cabré, 2006; Camacho, 2010; Aldenstein y Freixa, 2011; Borhn, 2015).
\end{abstract}

Palabras clave: diccionarización, productividad anual, productividad por diario, frecuencia de ocurre neológica, productividad por recurso de formación 
La neología política peruana en el nodo UNIFE del Observatorio de Neología...

Rosa Luna García

\begin{abstract}
The main objective of this empirical, applied, documentary and systematic terminographic study was to determine the productivity of Peruvian political neologisms collected in the Peruvian node UNIFE of the Observatory of Neology of the Pompeu Fabra University during the period 2003-2019. The research arrived at the following general conclusions: 1) The low number of tokens (repetitions) in the Peruvian political neological corpus could be attributed to problems in asystematic detection, to the mobility of the neologisms that integrate the Antenas Neológicas Unifé node or to the variability of neological sentiment. The authors Adelstein and Freixa (2013) argued in a research carried out from data provided by the Neology Observatory of the Pompeu Fabra University that these platforms are characterized by the presence of a high number of neologisms with low stabilization. 2) Regarding the annual productivity of political neologisms, a fairly balanced trend was observed between the two octennia of study. The first octenary of the study corpus (2004-2011) reached $42.2 \%$ compared to $57.6 \%$ of the second, with a $15.2 \%$ difference between them. 3) It was found that the criteria used for the dictionarization of neologisms in general language dictionaries (exclusion of local neologisms, ephemeral, derived from proper names and words with transparent productive suffixes [Aldenstein and Freixa, 2013]), differed from those related to the geolectal nature. Several authors have shown that in the latter, factors such as the witness character of new words in a given political period (Matoré, 1953; Aldenstein and Freixa, 2013) or the need to name a new word (Barnhart, 2007), 4) The formal newspapers El Comercio and La República were the most productive in Peruvian political neology, basically concentrated in the political, current affairs, national, editorial page and columns sections. 5) In the coining of Peruvian political neologisms, it was observed that derivation, suffixation and prefixation, together with composition (patrimonial and cultured) reached a high $81.7 \%$. This result is confirmed in several works on political neology (Díaz, 2001; Cabré, 2006; Camacho, 2010; Aldenstein and Freixa, 2011; Borhn, 2015).
\end{abstract}

Keywords: dictionaryization, annual productivity, productivity per journal, frequency of neological occurrence, productivity per training resource.

\title{
1. Introducción
}

\subsection{Planteamiento del problema}

\subsubsection{Formulación e importancia}

La Escuela de Traducción e Interpretación viene participando en el proyecto Antenas Neológicas desde el año 2003 y hasta la fecha ha recogido un total de 17 500 neologismos. A lo largo de estos años el equipo de trabajo ha ido cambiando, se ha contado con la participación de docentes, estudiantes y egresados de la Escuela de Traducción e Interpretación de la Facultad de Traducción, Interpretación y Ciencias de la Comunicación quienes recibieron capacitación y seguimiento 
por parte de la coordinadora del proyecto encargada de validar los candidatos a neologismos.

Los neologismos recogidos en las fichas neológicas de la plataforma OBNEO han sido extraídos de diarios y revistas peruanos de gran difusión y pertenecen a campos temáticos muy heterogéneos, a saber, tecnología, espectáculos, deportes, música, política local e internacional, divulgación médica, salud familiar, cultura, turismo, gastronomía, empresas, automovilismo, fianzas, cine, moda, cine, televisión, entre muchos otros.

Luego de revisar con detenimiento los neologismos de cada uno de estos campos, se optó por seleccionar el campo político nacional por ser uno de los que presenta mayor riqueza neológica.

A. Problema general

- ¿Qué productividad presentan los neologismos políticos peruanos incorporados en el observatorio de neología?

\section{B. Problemas específicos}

- ¿Cuál es la frecuencia de aparición de los neologismos políticos peruanos recogidos en la plataforma Obneo?

- ¿Cuál es la productividad anual por diario peruano?

- ¿Qué grado de diccionarización presenta el corpus de neologismos políticos?

- ¿Qué recursos de formación se emplearon para la acuñación de neologismos políticos peruanos?

\subsubsection{Importancia}

La importancia de este trabajo radica en que permitirá instaurar una nueva línea de investigación en la universidad: la neología. Los datos compilados en la plataforma OBNEO pueden ser explotados en diversas vertientes temáticas, a saber, para la redacción de artículos científicos, elaboración de tesis de bachiller y licenciatura, publicación de libros al igual que de diccionarios sobre neología peruana. 
La neología política peruana en el nodo UNIFE del Observatorio de Neología...

Rosa Luna García

\subsubsection{Antecedentes}

En el nivel local existe un antecedente indirecto que aborda la incidencia neológica en el nodo peruano de Antenas Neológicas en el que figuran neologismos políticos, entre muchos otros aspectos (Tavara y Vera, 2010), y uno directo (Luna y Soriano, 2010) que trata sobre neología peruana, específicamente derivada de los nombres propios Vladimiro Montesinos y Alberto Fujimori extraídos tanto del observatorio de neología como de otras fuentes periodísticas consultadas en línea.

En el nivel internacional hispano son tres las fuentes que contemplan el tema entre los años 2006 y 2020. El autor Vilches (2006) trabajó sobre la neología en el lenguaje político de manera general, Tomescu (2008) describió la neología política deonomástica (relativa a los nombres propios) y, finalmente, Pascual (2015) desarrolló el tópico de la tipologización y la creatividad en la neología política.

\subsubsection{Marco conceptual y teórico}

Para la construcción del marco teórico se tomaron en cuenta los trabajos sobre observatorios de neología de la Pompeu Fabra, el proyecto antenas neológicas, las investigaciones sobre metáforas políticas, nombres propios en el lenguaje político, recursos de formación neológica, y campos temáticos en neología política.

\subsubsection{Objetivos}

A. General

- Describir la productividad de los neologismos políticos peruanos recogidos en el nodo peruano Unife del Observatorio de Neología de la Universidad Pompeu Fabra durante el periodo 2003-2019

\section{B. Específicos}

- Localizar los neologismos peruanos con mayor frecuencia de aparición.

- Determinar los neologismos políticos con mayor productividad anual.

- Establecer el grado de diccionarización de los neologismos políticos.

- Ubicar los diarios con mayor productividad neológica de corte político.

- Identificar los recursos de formación empleados para la acuñación de los neologismos políticos peruanos.

86 Lengua y Sociedad. Revista de Lingüística Teórica y Aplicada 


\section{LINEAMIENTOS METODOLÓGICOS}

\subsection{Categorías y subcategorías de análisis}

La productividad neológica fue analizada a partir de las cinco categorías y subcategorías de análisis que se presentan en la siguiente tabla.

Tabla 1. Matriz de categorías y subcategorías

\begin{tabular}{l|l}
\hline Categorías & Subcategorías \\
$\begin{array}{l}\text { Productividad por frecuencia } \\
\text { de aparición }\end{array}$ & $\begin{array}{l}\text { Elevada } \\
\text { Promedio } \\
\text { Baja }\end{array}$ \\
\hline Productividad anual & 2003 al 2019 \\
\hline Grado de diccionarización & $\begin{array}{l}\text { Elevado } \\
\text { Promedio } \\
\text { Bajo }\end{array}$ \\
\hline \multirow{2}{*}{ Productividad por diario } & $\begin{array}{l}\text { La República } \\
\text { El Comercio } \\
\text { Trome } \\
\text { Expreso } \\
\text { Perú 21 } \\
\text { Correo }\end{array}$ \\
\hline Productividad por recurso de & $\begin{array}{l}\text { Prefijación } \\
\text { Sufijación } \\
\text { Composición } \\
\text { Semántico }\end{array}$ \\
& $\begin{array}{l}\text { Acronimia } \\
\text { Sintagmación }\end{array}$ \\
\hline
\end{tabular}

\subsection{Tipo, método y diseño de investigación}

El estudio es de naturaleza empírico-descriptiva. El diseño específico de investigación es no experimental cualitativo y cuantitativo, concretamente transeccional descriptivo y exploratorio. 
La neología política peruana en el nodo UNIFE del Observatorio de Neología...

Rosa Luna García

\subsection{Delimitación del corpus de estudio}

Para la extracción de datos se utilizó como corpus de vaciado la plataforma del Observatorio de Neología que es una «Plataforma de trabajo de acceso restringido a los miembros del Observatori de Neologia (OBNEO) y de las redes Antenas Neológicas, NEOROC y NEOXOC. Permite introducir y consultar los datos de los neologismos recogidos en el vaciado de cada grupo» (https://www.upf.edu/web/ obneo/recursos) en la que se encuentra el nodo peruano UNIFE del proyecto Antenas Neológicas a cargo de quien suscribe esta investigación.

De esta base de datos neológicos, se filtraron únicamente los neologismos de naturaleza política de procedencia peruana todos los cuales fueron ingresados por integrantes del grupo de terminología de la Universidad Femenina del Sagrado Corazón (UNIFETERM) durante los años 2003 al 2019 y extraídos de las secciones Política, Editorial, Actualidad, Local, Economía, Opinión, Columnas, Portada/ contraportada de un corpus de vaciado constituido por seis diarios peruanos $(E l$ Comercio, Perú 21, Correo, Expreso, Trome y La República).

El corpus de trabajo estuvo conformado por un total de 730 token (neologismos repetidos) con 302 types (palabras nuevas diferentes) que fueron objeto del análisis cuantitativo y cualitativo.

\subsection{Instrumento de recolección de datos y técnicas de procesamiento}

Luego de la extracción manual de neologismos políticos peruanos, se vaciaron los datos a una matriz con las categorías y subcategorías de análisis para su ulterior codificación y tabulación mediante tablas de estadística descriptiva se utilizó el software sPss.

\section{Resultados}

\subsection{Productividad por frecuencia de aparición}

La frecuencia de aparición de los neologismos políticos peruanos se analizó a partir de tres niveles de recurrencia: elevada (27-70 tokens), promedio (11-22 tokens) y baja (1-10 tokens). En la Tabla 2 presentaremos la frecuencia de ocurrencia propiamente dicha y en la Tabla 3 un ejemplo representativo de cada uno de los niveles.

88 Lengua y Sociedad. Revista de Lingüística Teórica y Aplicada 
La neología política peruana en el nodo UNIFE del Observatorio de Neología...

Rosa Luna García

\section{Tabla 2. Productividad neológica}

\begin{tabular}{|c|c|}
\hline FRECUENCIA & $\%$ \\
\hline 1 & 36 \\
\hline 2 & 16 \\
\hline 3 & 9 \\
\hline 4 & 6 \\
\hline 5 & 4 \\
\hline 6 & 3 \\
\hline 7 & 3 \\
\hline 8 & 2 \\
\hline 9 & 3 \\
\hline 10 & 1 \\
\hline 11 & 2 \\
\hline 12 & 3 \\
\hline 13 & 0,9 \\
\hline 14 & 0,5 \\
\hline 15 & 0,8 \\
\hline 16 & 0,8 \\
\hline 18 & 0,8 \\
\hline 19 & 0,8 \\
\hline 20 & 0,6 \\
\hline 22 & 0,8 \\
\hline 25 & 0,5 \\
\hline 27 & 0,5 \\
\hline 28 & 0,5 \\
\hline 31 & 0,5 \\
\hline 32 & 0,5 \\
\hline 43 & 0,5 \\
\hline 47 & 0,5 \\
\hline 48 & 0,5 \\
\hline 53 & 0,5 \\
\hline 55 & 0,5 \\
\hline 56 & 0,5 \\
\hline \multirow[t]{2}{*}{70} & 0,5 \\
\hline & 100 \\
\hline
\end{tabular}


La neología política peruana en el nodo UNIFE del Observatorio de Neología...

Rosa Luna García

A partir de los datos incluidos en la Tabla 2, se desprendió que la productividad neológica de los neologismos extraídos de OBNEO figuraba en el rango más bajo en tanto que los neologismos repetidos entre 1 y 10 veces, alcanzaron un considerable $83 \%$ del total frente a aquellos cuya recurrencia fue promedio, entre $11 \mathrm{y}$ 22 tokens (11\%) o baja, entre 25 y 70 ocurrencias (6 \%).

Tabla 3. Ejemplos de neologismos por frecuencia

\begin{tabular}{|l|l|}
\hline FRECUENCIAS & EJEMPLO \\
\hline 1 & fujicóctel \\
\hline 2 & acuerdista \\
\hline 3 & avenger \\
\hline 4 & estar chihuán \\
\hline 5 & acuñista \\
\hline 6 & primer damo \\
\hline 7 & narcocandidato \\
\hline 8 & fujiaprista \\
\hline 9 & faenón \\
\hline 10 & pulpín \\
\hline 11 & kenjista \\
\hline 12 & chavarrista \\
\hline 13 & chavarrizar \\
\hline 14 & fujimontesinista \\
\hline 15 & fujimontesinismo \\
\hline 16 & premierato \\
\hline 18 & naranja \\
\hline 19 & etnocacerista \\
\hline 20 & keikista \\
\hline 22 & desapristizar \\
\hline 25 & antifujimorista \\
\hline 27 & montesinista \\
\hline 28 & reeleccionista \\
\hline 31 & humalista \\
\hline 32 & megacorruptor \\
\hline 38 & narcoestado \\
\hline 43 & bicameralidad \\
\hline & \\
\hline &
\end{tabular}


La neología política peruana en el nodo UNIFE del Observatorio de Neología...

Rosa Luna García

\begin{tabular}{|l|l|}
\hline FRECUENCIAS & EJEMPLO \\
\hline 47 & megaproyecto \\
\hline 48 & narcoterrorismo \\
\hline 53 & fujimorismo \\
\hline 55 & multipartidario \\
\hline 56 & narcoterrorista \\
\hline 70 & narcoindulto \\
\hline
\end{tabular}

En los dos extremos de la Tabla 3 se ubicaron los neologismos fujicóctel y estar chihuán como ejemplos de baja recurrencia, con 1 y 4 respectivamente, y los nuevos vocablos narcoindulto y narcoterrorismo como evidencias de elevada repetición que variaron entre 48 y 70 tokens en la base oBNEO.

Entre los neologismos con recurrencia media se localizó a la dupla fujimontesinista- fujimontenisinismo que contó, respectivamente, con entre 14 y 15 repeticiones.

\subsection{Productividad neológica anual}

\section{Gráfico 1. Productividad neológica por año}

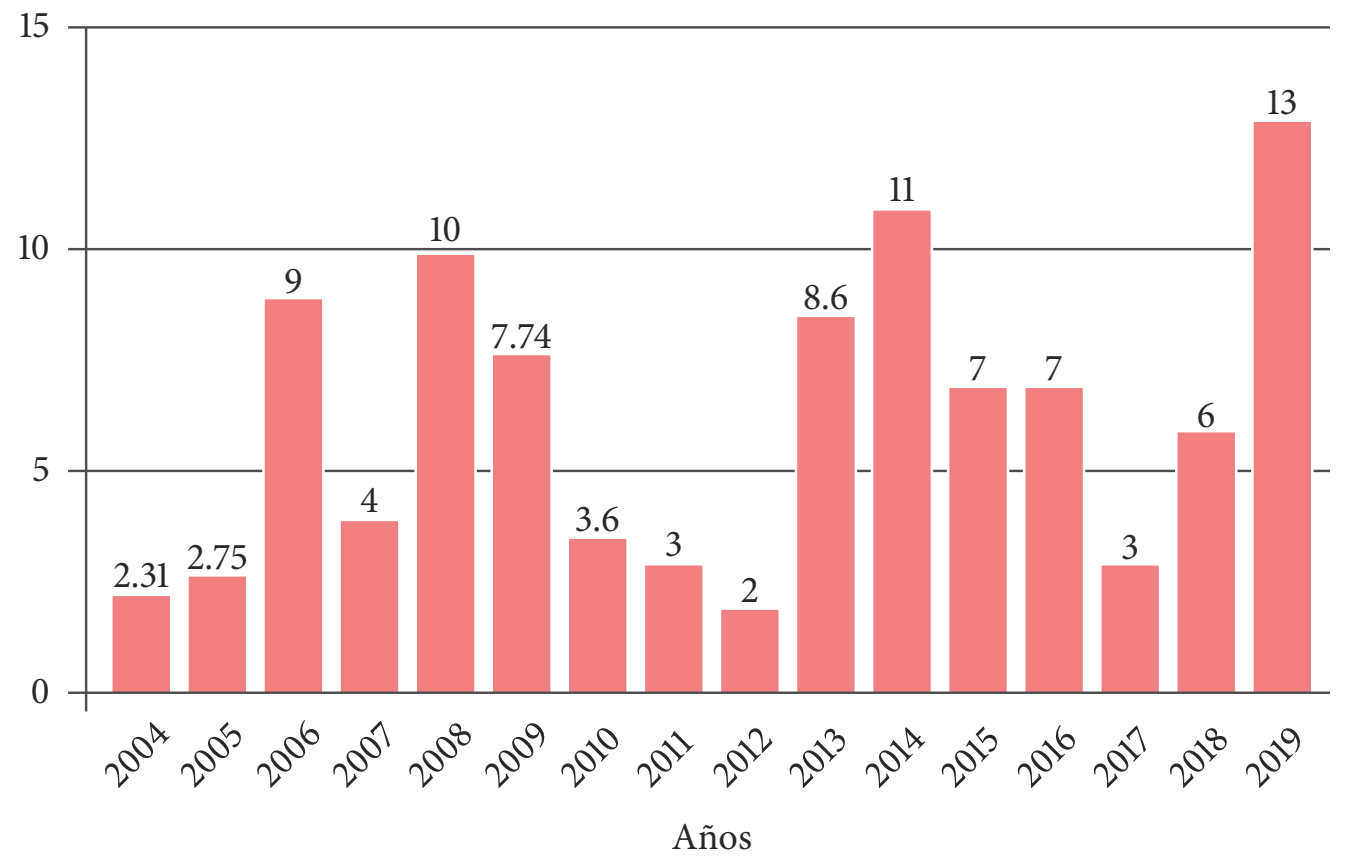


La neología política peruana en el nodo UNIFE del Observatorio de Neología...

Rosa Luna García

El Gráfico 1 evidenció la existencia de una productividad anual bastante dispar. Los años 2006, 2008, 2013 y 2014 fueron los más productivos, seguidos por seguidos por el 2009, 2015, 2016 y 2018 con un rendimiento medio, y finalmente 2004, 2005, 2007, 2010, 2011 y 2012 fueron los de menor productividad.

\subsection{Diccionarización}

\section{Gráfico 2. Porcentaje de diccionarización}

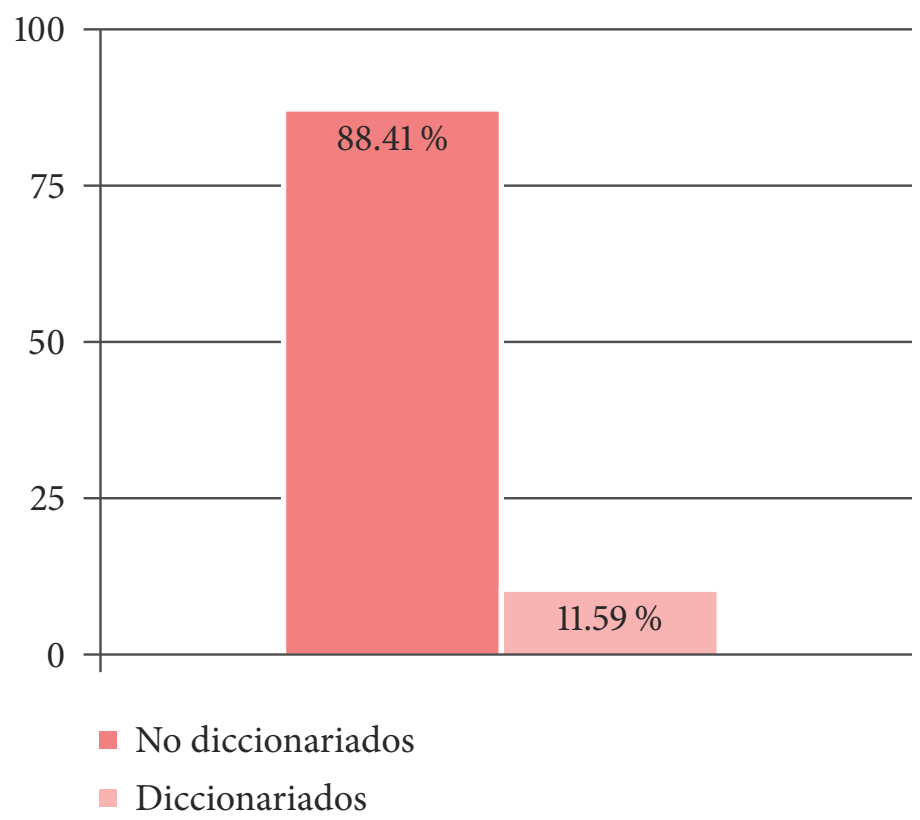

Tal como podrá observarse en el Gráfico 2, los neologismos políticos diccionarizados, esto es aquellos que pasaron a formar parte de los diccionarios de peruanismos, logró un bajo $12 \%$, frente a un elevadísimo 88,41 de vocablos nuevos que no fueron incorporados en los diccionarios. 


\section{Gráfico 3. Diccionarización por tipo de diccionarios}

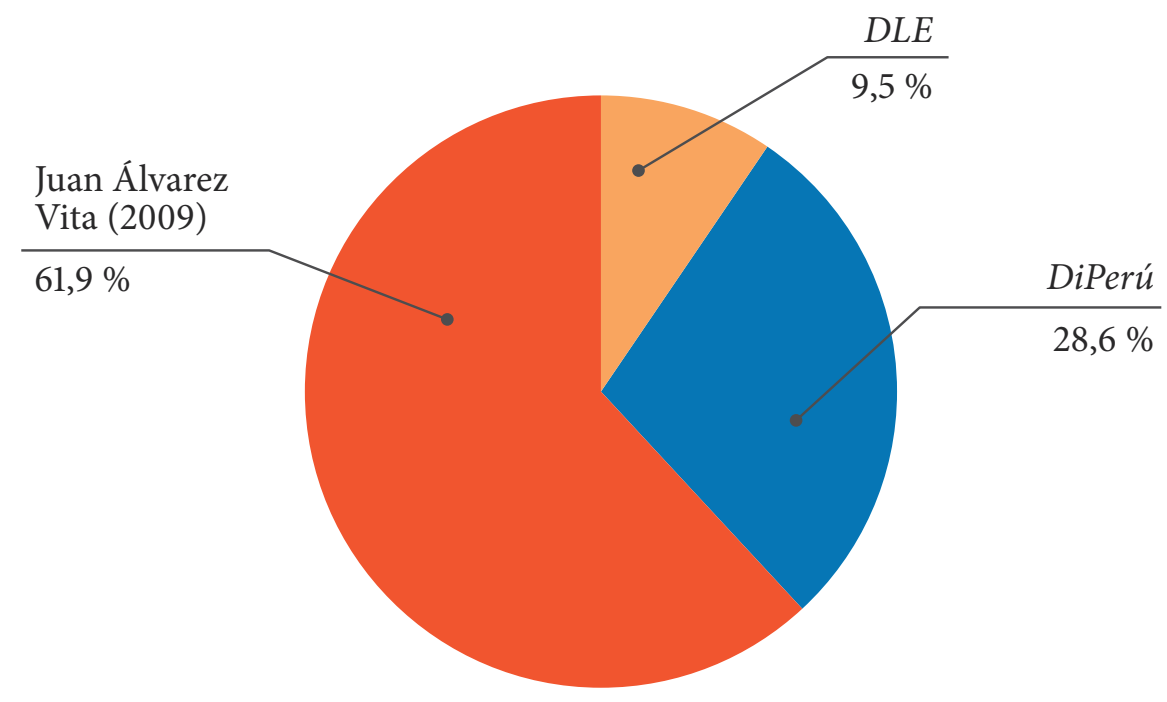

En términos de diccionarización por tipo de diccionario, los resultados arrojaron (Gráfico 3) que un elevado 61,9\% de los neologismos diccionariados se incorporaron en el Diccionario de Peruanismos (Álvarez, 2009), a 33 \% de diferencia con DiPerú (Calvo, 2016) y que el menor porcentaje (9,5\%) lo alcanzó el Diccionario de la Lengua Española (s. f.).

\section{Gráfico 4. Diccionarización por número de diccionarios}

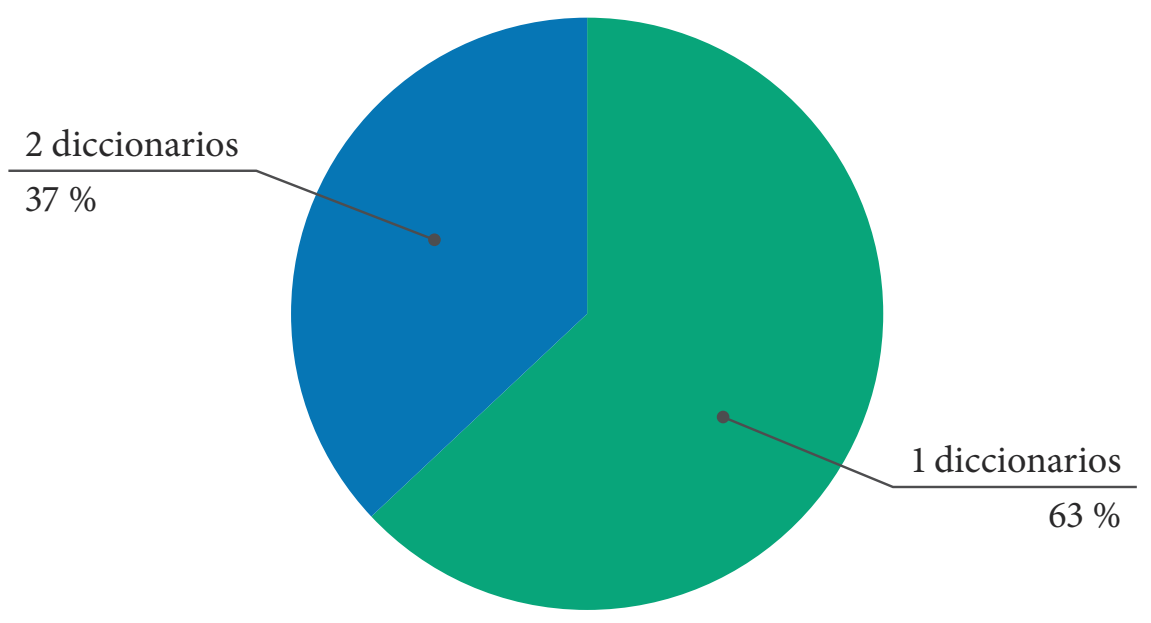


La neología política peruana en el nodo UNIFE del Observatorio de Neología...

Rosa Luna García

Complementariamente, el Gráfico 4 mostró de manera contundente que el $37 \%$ de neologismos políticos figuraba únicamente en dos diccionarios de peruanismos (Álvarez, 2009; y DiPerú, 2016) y un elevado $63 \%$ solo en una de las tres fuentes del corpus de exclusión.

Cabe destacar que no hubo ningún neologismo recogido en las tres fuentes del corpus de exclusión utilizado para este estudio.

Tabla 4. Neologismos diccionariados en corpus de exclusión

\begin{tabular}{|c|c|c|c|}
\hline Neologismo & DLE (2014) & DP (2015) & JAV (2009) \\
\hline alanismo & & 1 & \\
\hline alanista & & 1 & 1 \\
\hline chacanista/chakanista & & & 1 \\
\hline chakana & & & 1 \\
\hline clientela & 1 & & \\
\hline clientelista & 1 & & \\
\hline copamiento & & 1 & \\
\hline etnocacerismo & & 1 & 1 \\
\hline etnocacerista & & 1 & 1 \\
\hline faenón & & 1 & \\
\hline fimista & & & 1 \\
\hline fujimontesinismo & & 1 & 1 \\
\hline fujimontesinista & & 1 & 1 \\
\hline fujimorato & & 1 & 1 \\
\hline fujimorismo & & 1 & 1 \\
\hline fujimorista & & 1 & 1 \\
\hline fujimorada & & 1 & 1 \\
\hline humalismo & & & 1 \\
\hline humalista & & & 1 \\
\hline izquierda caviar & & 1 & \\
\hline nacionalismo & & 1 & \\
\hline nacionalista & & 1 & \\
\hline otorongo/ga & & 1 & 1 \\
\hline otoronguismo & & 1 & 1 \\
\hline peruposibilismo & & & 1 \\
\hline
\end{tabular}


La neología política peruana en el nodo UNIFE del Observatorio de Neología...

Rosa Luna García

\begin{tabular}{l|l|l|l}
\hline Neologismo & DLE (2014) & DP (2015) & JAV (2009) \\
\hline perubosibilista & & 1 & 1 \\
\hline policastro & $\begin{array}{l}\text { politicastro } \\
\text {-tra }\end{array}$ & & 1 \\
\hline toledato & & & 1 \\
\hline toledismo & & & 1 \\
\hline toledista & & & 1 \\
\hline upepismo & & & 1 \\
\hline upepista & & & 1 \\
\hline vladivideo & & & 1 \\
\hline
\end{tabular}

En la Tabla 4, se ilustran los 33 neologismos políticos recopilados en los diccionarios del corpus de exclusión que equivalieron al $11 \%$ del total de los ingresados por el nodo peruano de Antenas Neológicas a partir del año 2003.

\subsection{Productividad por diario}

\section{Gráfico 5. Productividad por diario}

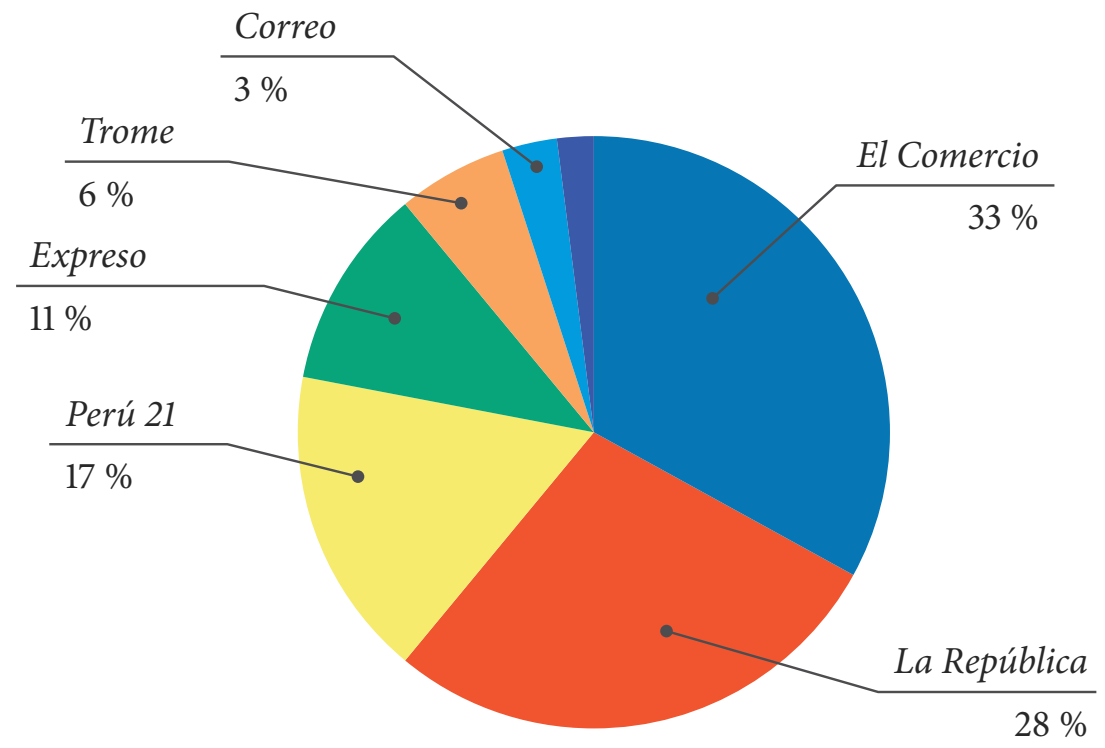


La neología política peruana en el nodo UNIFE del Observatorio de Neología...

Rosa Luna García

En la Figura 5 se constató que los diarios El Comercio, La República y Perú 21 ocuparon el primer lugar en cuanto a productividad neológica con $33 \%$, 28 \% y $17 \%$ respectivamente. En el otro extremo se encontraron los diarios con menor neologicidad política: Trome con un escaso 6 \% y Correo con un ínfimo 3 \%.

\subsection{Productividad por recurso de formación}

\section{Gráfico 6. Productividad por recurso de formación}

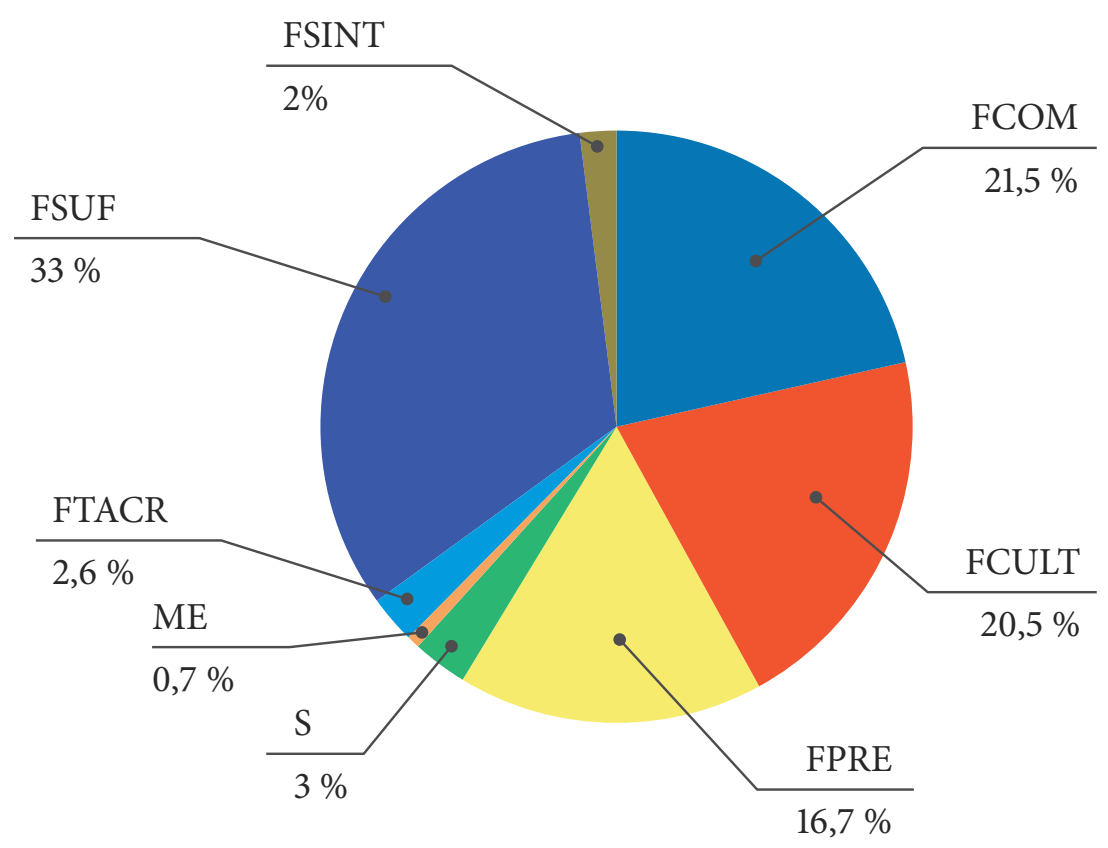

A partir del Gráfico 6 puede colegirse que los recursos de formación más recurrentes en la neología política peruana estudiada fueron la sufijación con $33 \%$, acompañado de cerca por la composición patrimonial y culta con porcentajes muy estrechos (21,5 y 20,5), a escasa distancia de la prefijación que obtuvo un $16,7 \%$. Los recursos menos utilizados fueron el semántico (3\%), la acronimia $(2,6 \%)$, la sintagmación ( $2 \%$ y el préstamo del inglés $(0,7 \%)$.

96 Lengua y Sociedad. Revista de Lingüística Teórica y Aplicada 


\section{Discusión}

\subsection{Frecuencia de ocurrencia:}

El escaso número de tokens (repeticiones) del corpus neológico político peruano podría atribuirse a problemas en la detección asistemática, a la movilidad de los neólogos que integran el nodo de Antenas Neológicas Unifé o al sentimiento neológico variable. Las autoras Adelstein y Freixa (2013) argumentaron en su investigación a partir de datos proporcionados por el Observatorio de Neología de la Universidad Pompeu Fabra que estas plataformas se caracterizan por la presencia de un elevado número de neologismos con baja estabilización. Los datos que ellas obtuvieron del Observatorio de Neología de la Universidad Pomepu Fabra mostraron un elevadísimo 78,6 \% con una sola ocurrencia y un 10,68 \% con apenas dos apariciones.

\subsection{Productividad anual:}

En lo que respecta a la productividad anual de neologismos políticos se observó, a pesar de la disparidad por año, una tendencia bastante equilibrada entre los dos octenios de estudio. El primer octenio del corpus de estudio (2004-2011) alcanzó un $42,2 \%$ frente a un 57,6 \% del segundo, con un $15,2 \%$ de diferencia entre ellos.

\subsection{Diccionarización:}

Uno de los criterios válidos para evaluar el grado de diccionarización es sin duda la antigüedad del corpus de exclusión. La fuente lexicográfica que cuenta con mayor porcentaje de neologismos está bastante desactualizada (Álvarez, 2009) y que el diccionario más reciente (DiPerú, 2016) solo diccionariza un $16 \%$ adicional de neologismos políticos.

En lo tocante a los criterios utilizados para la diccionarización de neologismos en diccionarios de lengua general (exclusión de neologismos locales, efímeros, derivados de nombres propios), se confirmó que estos difieren de empleados por los geolectales. En trabajos neológicos de corte geolectal prima el carácter testigo de los nuevos vocablos (Matoré, 1953) y la necesidad de denominar la palabra nueva (Barnhart, 2007). En esta misma línea de pensamiento, para las neológas Adelstein y Freixa (2013) «la no calculabilidad, muchas veces ligada al carácter testigo de la unidad, que constituye un factor importante a tener en cuenta a la hora de decidir el ingreso de neologismos en el diccionario» (p. 11) y añaden 
que «la neología formal creada a partir de sufijos productivos no suele incluirse en diccionarios de lengua general, sin embargo, los neologismos testigos de un período político sí son ingresados en diccionarios regionales» (2013, p. 13).

\subsection{Productividad por diario}

Los diarios formales El Comercio y La República fueron los más productivos en neología política peruana, concretamente las secciones política, actualidad, nacional, página editorial y columnas.

\subsection{Recursos de formación léxica:}

En la acuñación de neologismos políticos peruanos se observó que la derivación, sufijación y prefijación, conjuntamente con la composición (patrimonial y culta) alcanzaron un elevado 81,7\%. El grueso de neologismos políticos recopilados (70 \%) derivaron de nombres propios de políticos o de partidos políticos (epónimos). Este resultado se confirma en diversos trabajos sobre neología política (Díaz, 2001; Cabré, 2006; Camacho, 2010; Adelstein y Freixa, 2013; Borhn, 2015). 


\section{Referencias bibliográficas}

Adelstein, A. y Freixa, J. (2013, 28-30 de octubre). Criterios para la actualización lexicográfica a partir de datos de observatorios de neología [Presentación de escrito]. Congreso Internacional El Diccionario: neología, lenguaje de especialidad, computación, Ciudad de México. https://repositori.upf.edu/ handle/10230/34891 ?locale-attribute=es

Álvarez, J. (2009). Diccionario de peruanismos. El habla castellana en el Perú. Universidad Alas Peruanas.

Barnhart, D. K. (2007). A calculus for new words. Dictionaries, 28, 132-138.

Bernal, E.; Freixa, J. y S. Torner (2018). Frecuencia, estabilidad... ¿y después qué? Criterios para la diccionarización de neologismo. https://www.upf.edu/web/ neometro/frecuencia

Bohrn, A. (2015). Neologismos derivados de nombres propios en el español de la Argentina. En I. Alves y E. Simões (Eds.), Neologia das Línguas Românicas (pp. 547-562). Humanitas.

Cabré, M. T. (2006). La clasificación de neologismos: una tarea compleja. Alfa Revista de Linguística, 50(2), 229-250.

Calvo, J. (Dir.). (2016). DiPerú. Diccionario de peruanismos. Academia Peruana de la Lengua; Minera Buenaventura.

Camacho, J. C. (2010). El nombre propio en la neología. Deonomástica y Eponimia. En M. T. Cabré, O. Domènech, R. Estopà, J. Freixa y M. Lorente (Coords.), Actes del I Congrés Internacional de Neología en les llengües Romàniques. Universitat Pompeu Fabra; Institut Universitari de Lingüística Aplicada.

Díaz, J. A. (2001). Nociones de neología. La formación de derivados y compuestos a partir de nombres propios de personas.Panace@, 2(5).25-30.

Luna, R. y Soriano, R. (2010). Fujimorización y vladimirización de la neología peruana. En Actas deI II Congreso Internacional de Neología en Lenguas Neolatinas. Barcelona, España.

Matoré, G. (1953). La méthode en lexicologie. Marcel Didier.

Pascual, M. (2015). Neología y prensa. Neologismos en el periodismo político. Análisis en torno a la tipología y la creatividad [Trabajo de fin de grado, Universidad Católica de San Antonio]. RIUCAM UCAM Institutional Repository. http://repositorio.ucam.edu/handle/10952/1416

Real Academia Española. (s. f.). Diccionario de la lengua española (23. ${ }^{\text {a }}$ ed.) [Versión 23.4 en línea]. https://dle.rae.es 
La neología política peruana en el nodo UNIFE del Observatorio de Neología...

Rosa Luna García

Tavara, S. y Vera, S. (2010). Incidencia de neologismos en el nodo peruano de antenas neológicas [Tesis de licenciatura, Universidad Femenina del Sagrado Corazón - Unifé]. Base de Datos Biblio de la Universidad Femenina del Sagrado Corazón - Unifé.

Tomescu, E. (2008,7-10 de mayo). Les neologismes denonomastoiques [Presentación de cartel]. I Congreso Internacional de Neología en las lenguas románicas (CINEO 2008), Barcelona, España.

Vilches, F. (2006). El neologismo en el lenguaje político. En Autor (Coord.), Creación neológica y las nuevas tecnologías (pp. 259-310). Dykinson.

\section{Trayectoria académica de la autora}

Rosa Luna García es doctora en Educación (UNIFÉ), magíster en Docencia Universitaria (UNIfÉ), magíster en Terminología por la Universidad Pompeu Fabra y en Docencia Universitaria por la UNIFÉ; segunda especialidad en Gestión y Didáctica de Programas de Educación a Distancia por la Universidad Católica del Perú (PUCP, 2006), licenciada en Traducción (URP), docente e investigadora de las Escuelas Profesionales de Traducción e Interpretación (UnIfÉ y Universidad Ricardo Palma), coordinadora del proyecto Antenas Neológicas (Universidad Pompeu Fabra-UnifÉ). Es autora del libro Temas de traducción (UNIFÉ, ediciones 2000 y 2002); del Diccionario para profesionales de la traducción (Universidad Peruana de Ciencias Aplicadas, 2017, en coautoría con Mary Ann Monteagudo); Diccionario de la educación e inclusión lingüística peruana (Universidad Femenina del Sagrado Corazón, 2019, en coautoría con Claudia Sánchez); Diccionario de Bullying y Diccionario de Violencia contra la Mujer (Universidad Ricardo Palma, 2020). Exvicepresidenta de la Red Iberoamericana de Terminología, y actual miembro de la Junta de Vigilancia de Traductores (Ministerio de Relaciones Exteriores). Sus campos de investigación son la terminología, la neología y la traductología. 